\title{
Design and Analysis of Transceiver for Combating Turbulence Induced Fading over Fso Links
}

\author{
R.Somasundaram ${ }^{1}$, R.Marthandan ${ }^{2}$ \\ I' PG Scholar in Communication Systems, Adhiyamaan College Of Engineering, Hosur,India) \\ ${ }^{2}$ ( AP in Electronics And Communication Engineering, Adhiyamaan College Of Engineering, Hosur,India)
}

\begin{abstract}
In this paper, we investigate the cooperative diversity technique as a candidate solution for combating turbulence induced fading over Free-Space Optical (FSO) links. In particular, a one-relay cooperative diversity scheme is proposed and analyzed for non-coherent FSO communications with intensity modulation and direct detection (IM/DD). The error performance is derived in semi-analytical and closed-form expressions in the presence and absence of background radiation, respectively. Results show the enhanced diversity orders that can be achieved over both Rayleigh and lognormal fading models. In this transceiver design coherent transmitter and receiver design is carried out with the reference to q-ary PPM(pulse position modulation).performance analysis is carried out in all possible strategy.
\end{abstract}

Keywords: Free-space optics, spatial diversity, cooperative diversity, atmospheric turbulence.

\section{INTRODUCTION}

FREE-SPACE OPTICAL (FSO) communications attracted significant attention as a promising solution for the "last mile" problem [1]. A major impairment that severely degrades the link performance is fading (or scintillation) that results from the variations of the index of refraction due to in homogeneities in temperature and pressure changes [2]. In order to maintain acceptable performance levels over FSO links, fading-mitigation techniques such as spatial diversity techniques must be employed. Spatial diversity involves the deployment of multiple transmit and/or receiver apertures and is commonly used to combat fading and improve the link reliability. Diversity combining techniques were extensively studied in the context of radio-frequency (RF) wireless transmissions and were recently extended and tailored to FSO transmissions. In this context, apertureaveraging receiver diversity [3], spatial repetition codes [4], unipolar versions of the orthogonal space-time codes [5] and transmit laser selection [6] were proposed as FSO-adapted spatial diversity solutions. In the same way, the bit error rates of Multiple-Input-Multiple-Output (MIMO) FSO links were evaluated in [7] and [8]. On the other hand, in RF systems cooperative diversity techniques are becoming more popular in situations where limited number of antennas can be deployed at the mobile terminals [9], [10]. In this context, neighbouring nodes can form "virtual" antenna arrays and profit from the underlying spatial diversity in a distributed manner. Cooperative diversity takes advantage of the broadcast nature of RF transmissions where a message transmitted from a source to a destination can be overheard by neighbouring nodes. If these nodes are willing to cooperate with the source, they retransmit information about the same message to the destination thus enhancing the quality of signal reception. Despite the extensive research in RF wireless cooperation, to the authors' best knowledge, this technique was never considered before in the context of FSO communications. In this paper, we investigate for the first time the utility of cooperative diversity as a means of combating fading in FSO links. In particular, we consider a decode-and-forward strategy with one relay over FSO links with intensity modulation and direct detection (IM/DD). The main reason that discourages the investigation of cooperation in FSO systems resides in the non-broadcast nature of optical transmissions. In FSO, the source can not broadcast an information message to the destination and to a distant relay simultaneously. Consequently, an additional transmit power must be entirely dedicated for delivering the message to the relay. None of this power will reach the destination implying an additional power penalty (as compared to RF wireless cooperation). However, this work shows that the cooperative diversity can be useful in enhancing the performance of FSO links since the gain in diversity can compensate for this power penalty. On the other hand, the factors encouraging the implementation of the cooperative diversity technique are as follows. (1): The solution is cost-effective (compared to MIMO-FSO) since it does not require adding more apertures to the transmitter and/or receiver. (2): It is well known that channel correlation degrades the performance of MIMO systems whether in FSO or RF scenarios. However, MIMO-FSO channels are more likely to be correlated. In fact, in RF systems the signal reaches the receiver by a large number of paths implying that a small separation between the antennas can ensure a channel independence. On the other hand, FSO links are much more directive thus rendering the path gains between the transmit and receive elements more dependent; for example, the presence of a small cloud between the transmitter and receiver can induce large fades on all source-detector pairs simultaneously [4]. Consequently, the high performance gains promised by MIMO-FSO solutions under the assumption of channel independence 
[4]-[6] might not be achieved in practice. Consequently, as stated in [4] "alternative means of operation in such environments must be considered". In this context, cooperation can constitute a good candidate alternative. In fact, given the large distances (in the order of kilometres) between the source-destination, source-relay and relay-destination, the assumption of independence is more valid compared to the case of co-located arrays. (3): Unlike RF systems, extending the MIMO techniques to FSO systems imposes a compromise on the choice of the sources. The sources must be narrow enough to couple more power from the transmitter to the receiver and they must be wide enough to illuminate all detectors simultaneously. Consequently, the wider spacing of detectors (necessary for channel independence) results in an increased transmit power. In this context, taking advantage of the spatial diversity in a distributed manner by deploying the proposed cooperative scheme permits to overcome this limitation and can constitute a good practical alternative to MIMO-FSO systems.

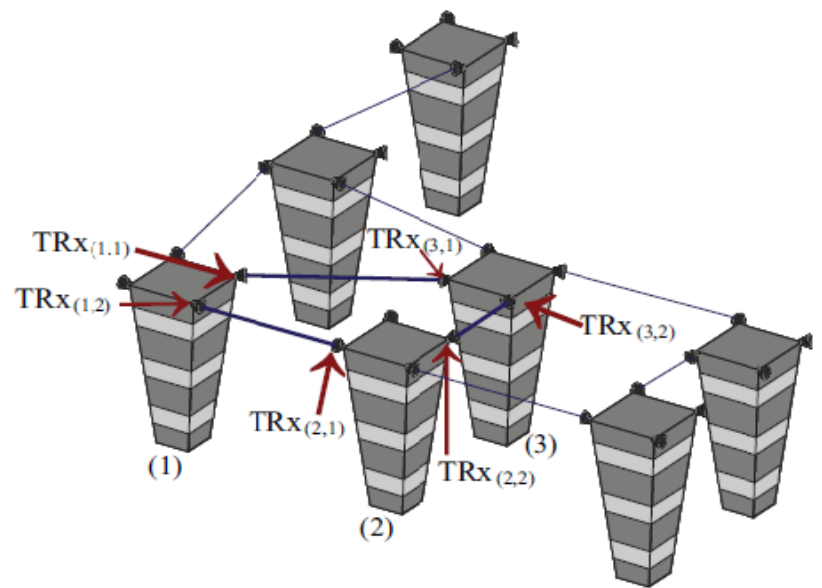

Figure 1. An example of a mesh FSO network. Cooperation is proposed among the transceivers on buildings (1), (2) and (3) where the transceivers on building 2 can help in transmitting an information message from (1) to (3). Note how, given the non-broadcast nature of FSO transmissions, one couple of FSO transceiver units is dedicated for each link.

\section{System Model And Transmitter Structure}

Consider the example of a FSO Metropolitan Area Network as shown in Fig. 1. Consider three neighbouring buildings (1), (2) and (3) and assume that a FSO connection is available between each building and its two neighbouring buildings. In FSO networks, each one of these connections is established via FSObased wireless units each consisting of an optical transceiver with a transmitter and a receiver to provide full duplex capability. Given the high directivity and non-broadcast nature of FSO transmissions, one separate transceiver is entirelydedicated for the communication with each neighbouring building. We assume that the transceivers on building (2) are available for cooperation to enhance the communication reliability between buildings (1) and (3). By abuse of notations, buildings (1), (2) and (3) will be denoted by source (S), relay (R) and destination (D), respectively. The cooperation strategy is depicted in Fig. 2. It is worth noting that the transceivers at (R) are not deployed with the objective of assisting (S). In fact, these transceivers are deployed for $(\mathrm{R})$ to communicate with $(\mathrm{S})$ and $(\mathrm{D})$; if $(\mathrm{R})$ is willing to share its existing resources (and (R) has no information to transmit), then it can act as a relay for assisting (S) in its communication with (D). The cooperation strategy is as follows: a sequenceof symbols is first transmitted to the relay. At a second time, (R) transmits the decoded symbols to (D) while (S) transmits the same symbol sequence simultaneously to (D). Since three transmissions are involved in each cooperation cycle, then the transmitted power from transceivers TRx $S, 1, \mathrm{TRx} S, 2$ and TRx $R, 2$ must be divided by 3 . Denote by $a 0, a 1$ and $a$ the random path gains between S-D, S$\mathrm{R}$ and R-D, respectively. In this work, we adopt the lognormal and Rayleigh turbulence-induced fading channel models [4]. In the lognormal model, the probability density function (pdf) of the path gain $(a>0)$ is given by: where the parameters $\mu$ and $\sigma$ satisfy the relation $\mu=-\sigma 2$ so that the mean path intensity is unity: $\mathrm{E}[I]$ $=\mathrm{E}\left[A_{2}\right]=1$. The degree of fading is measured by the scintillation index defined by: S.I. $=e^{4 \sigma^{2}}-1$. Typical

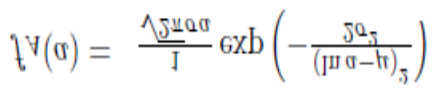

values of S.I. range between 0.4 and 1. In the Rayleigh model, the pdf of the path gain $(a>0)$ is: $(a)=2 a_{e}-a_{2}$. 
Consider $Q$-ary pulse position modulation (PPM) with IM/DD links where the receiver corresponds to a photoelectrons counter. Consider first the link S-D and denote by $(0)=[(0) 1, \ldots, Z(0) Q]$ the $Q$-dimensional vector whose $q$-th component $Z(0) q$ corresponds to the number of photoelectron counts in the $q$-th slot. Denote the transmitted symbol by $s \in\{1, \ldots$,$\} . The decision variable (0) s$ can be modelled as a Poisson random

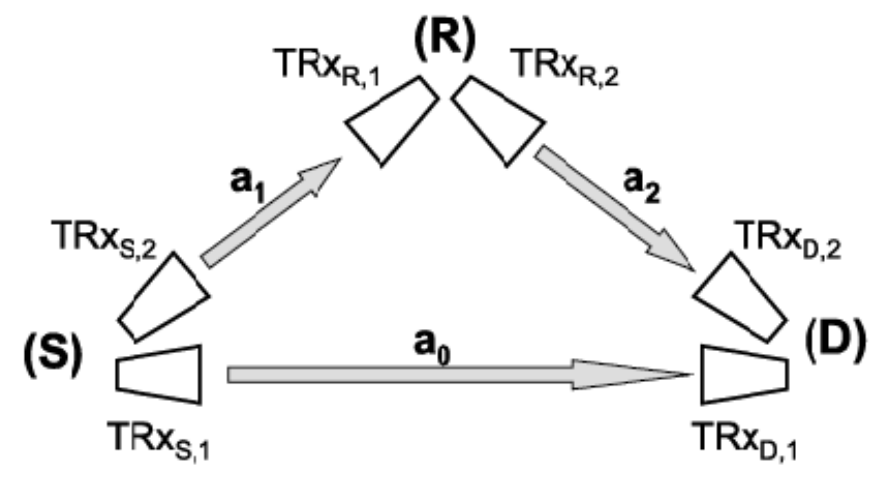

Fig. 2. The proposed cooperation scheme.

variable (r.v.) $q /=s$ ) can be modeled as a Poisson r.v. with parameter $\lambda n$ [4]:

Where $\lambda s$ (resp. $\lambda n$ ) corresponds to the average number of photoelectrons per slot due to the light signal (resp. Background radiation and "dark currents"):

$$
\begin{aligned}
& \operatorname{Pr}\left(Z_{q}^{(0)}=k\right)= \begin{cases}\frac{e^{-\left(\mu_{0}^{2} \lambda_{s} / 3+\lambda_{n}\right)}\left(a_{0}^{2} \lambda_{s} / 3+\lambda_{n}\right)^{\alpha}}{e^{\lambda_{n}} \lambda_{n}^{k}}, k ! & q=s ; \\
k ! & q \neq s .\end{cases} \\
& \lambda_{s}=\eta \frac{P_{r} T_{s} / Q}{h f}=\eta \frac{E_{s}}{h f} ; \lambda_{n}=\eta \frac{P_{b} T_{s} / Q}{h f}
\end{aligned}
$$

Where $\eta$ is the detector's quantum efficiency assumed to be equal to 1 in what follows, $h=6.6 \times 10-34$ is Planck's constant and $f$ is the optical centre frequency taken to be $1.94 \times 1014 \mathrm{~Hz}$ (corresponding to a wavelength of $1550 \mathrm{~nm}$ ). Ts stands for the symbol duration, $\operatorname{Pr}$ stands for the optical power that is incident on the receiver and $P b$ corresponds to the incident background power. Finally, Es $=P r T s / Q$ corresponds to the received optical energy per symbol corresponding to the direct link S-D. Readers can refer to [4] for more details on the system model. In the same way, we denote the decision vector corresponding to the S-R link by $Z(1)=[Z(1) 1, \ldots, Z(1) Q]$ where the parameter of the Poisson r.v. $Z(1) q$ is given by:

Where $\beta 1$ is a gain factor that follows from the fact that $(\mathrm{S})$ might be closer to (R) than it is to (D). In other

$$
\mathrm{E}\left[Z_{q}^{(1)}\right]= \begin{cases}\beta_{1} \frac{a_{1}^{2} \lambda_{s}}{3}+\lambda_{n}, & q=s \\ \lambda_{n}, & q \neq s .\end{cases}
$$

words, the received optical energy at (R) corresponding to $E s$ (that corresponds to the S-D link) is $\beta 1 E s$. Performing a typical link budget analysis [4] shows that $\beta_{1}=\left(\mathrm{d}_{\mathrm{SD}} / \mathrm{d}_{\mathrm{SR}}\right)^{2}$ where $d_{S D}$ and $d_{S R}$ stand for the distances from (S) to (D) and (S) to $(\mathrm{R})$ respectively. The maximum-likelihood (ML) decision rule at $(\mathrm{R})$ is given by: ${ }^{\wedge} s=\operatorname{argmax} q=1 \ldots . . Q(1)$. The relay transmits the symbol ^ $s$ along the link R-D implying that the corresponding decision vector can be written as $(2)=[Z(2) 1, \ldots, Z(2) Q]$

where $Z(2) q$ is a Poisson random variable with: $\beta_{2}=\left(\mathrm{d}_{\mathrm{SD}} / \mathrm{d}_{\mathrm{SR}}\right)^{2}$ with $d R D$ corresponding to the distance between (R) and (D). Finally, note that the normalization of $\lambda s$ by 3 in equations (1), (3) and (4) ensures that the total transmit power is the same as in non-cooperative systems.

$$
\mathrm{E}\left[Z_{q}^{(2)}\right]= \begin{cases}\beta_{2} \frac{a_{2}^{2} \lambda_{s}}{3}+\lambda_{n}, & q=\hat{s} \\ \lambda_{n}, & q \neq \hat{s} .\end{cases}
$$




\section{Receiver Structure}

As in all cooperative schemes, decoding will be based on the assumption: $p_{e} \triangleq \operatorname{Pr}\left({ }^{\wedge} s /=s\right) \ll 1$. This is justified since these schemes result in performance gains only for large values of $E s$.

\section{A. Detection in the Absence of Background Radiation}

In the absence of background radiation, (0) and (2) contain at least $Q-1$ empty slots each. In this case, the detection procedure at (D) is as follows. If one component of (0) is different from zero, this will imply that the symbol $s$ was transmitted in the corresponding slot since in the absence of background radiation the only source of this nonzero count is the presence of a light signal in this slot. On the other hand, if all components of (0) are equal to zero, then the decision will be based on (2). If one component of (2) is different from zero, then with probability $1-p_{e}$ this component corresponds to $s$ and with probability $p_{e}$ this component corresponds to an erroneous slot. Since $1-$ is assumed to be greater than $p_{e}$ (since $p_{e}<<1$ ), then the best strategy is to decide in favour of the nonempty slot of (2). Finally, if all components of (0) and (2) are equal to zero, then (D) decides randomly in favour of one of the $Q$ slots. To summarize, (D) decides in favour of $\sim \mathcal{s}$ according to the following strategy:

$$
\tilde{s}= \begin{cases}\arg _{q}\left[Z_{q}^{(0)} \neq 0\right], & Z^{(0)} \neq 0_{Q} ; \\ \arg _{q}\left[Z_{q}^{(2)} \neq 0\right], & Z^{(0)}=0_{Q}, Z^{(2)} \neq 0_{Q} ; \\ \operatorname{rand}(1, \ldots, Q), & Z^{(0)}=Z^{(2)}=0_{Q} .\end{cases}
$$

where $\mathbf{0}_{Q}$ corresponds to the $Q$-dimensional all-zero vector while the function $\operatorname{rand}(1, \ldots, Q)$ corresponds to choosing randomly one integer in the set $\{1, \ldots, Q\}$.

\section{B. Detection in the presence of background radiation}

In this case, the background radiation results in nonzero counts even in empty slots necessitating a more complicated detection procedure. The optimal ML detection procedure must take into consideration that ${ }^{\wedge} s$ might be different from $s$. Note that ${ }^{\wedge} s=s$ with probability $1-p_{e}$ while ${ }^{\wedge} s$ can correspond to a certain slot that is different from $s$ with probability $p_{d} Q^{-1}$. In this case, eliminating all common terms in the log-likelihood function, it can be proven that the ML decision rule is given by:

Even though optimal, the above decoder is not feasible for practical implementation since it requires the

$$
\begin{aligned}
& (\tilde{s}, \hat{s})=\underset{q, q^{\prime} \in\{1, \ldots, Q\}}{\arg \max _{q}}\left[Z_{q}^{(0)} \ln \left(1+\frac{a_{0}^{2} \lambda_{s}}{3 \lambda_{n}}\right)\right. \\
& \left.+Z_{q^{\prime}}^{(2)} \ln \left(1+\beta_{2} \frac{a_{2}^{2} \lambda_{s}}{3 \lambda_{n}}\right)\right]+ \begin{cases}\ln \left(1-p_{e}\right), & q=q^{\prime} ; \\
\ln \left(\frac{p_{e}}{Q 1}\right), & q \neq q^{\prime} .\end{cases}
\end{aligned}
$$

knowledge of $p e$ which is not available. On the other hand, given that $p e<<1$, then we can build a simpler decoder that is based on the assumption that the decision made at the relay is correct $\left({ }^{\wedge} s=s\right)$. In this case, the decision rule given in eq. (6) simplifies to:

Equation (7) corresponds to evaluating weighted sums of the photoelectron counts. An even simpler decision rule adopts equal weights and is given by:

$$
\tilde{s}=\underset{q \in\{1, \ldots, Q\}}{\arg \max }\left[Z_{q}^{(0)} \ln \left(1+\frac{a_{0}^{2} \lambda_{s}}{3 \lambda_{n}}\right)+Z_{q}^{(2)} \ln \left(1+\beta_{2} \frac{a_{2}^{2} \lambda_{s}}{3 \lambda_{n}}\right)\right]
$$

In this paper, we adopt the equal-gain combiner (EGC) described in eq. (8) for the following reasons. (i)

$$
\tilde{s}=\underset{q \in\{1, \ldots, Q\}}{\arg \max _{q}}\left[Z_{q}^{(0)}+Z_{q}^{(2)}\right]
$$

Simulations showed that the performance levels achieved by the decoders given in equations (6), (7) and (8) are very close to each other. In fact, for practical values of $E s$, the decoders in equations (6) and (7) are extremely close to each other (which is justified since $p_{e}<<1$ ) and their performance gain with respect to EGC is negligible. (ii) The implementation of EGC is much simpler since it does not require any form of training for acquiring the values of the channel gains as well as $\lambda s$ and $\lambda n$. Finally, equations (5) and (8) show that the proposed cooperation strategy can be implemented without requiring any channel state information neither at the transmitter nor at the receiver sides. 


\section{Numerical Results And Simulation Results}

We assume that $d_{S D}=d_{R D}$ implying that $\beta_{2}=1$. The performance of the $2 \times 1$ MIMO-FSO links in [4] that deploy repetition coding (RC) and that are capable of achieving a full transmit diversity order is also included as a benchmark. Fig. 3 shows the performance of 4-PPM in the absence of background radiation over Rayleigh fading channels. This figure shows the excellent match between simulations and the exact SEP expression in eq. (14). The slopes of the SEP curves indicate that cooperation results in an increased diversity order of two for various distances of (R) from (S). Even in the extreme case where (S) is as far from (R) as it is from (D) $(\beta 1=1)$, a gain of about $8 \mathrm{~dB}$ at a SEP of $10-3$ can be observed relative to non-cooperative systems. The excellent match between simulations and eq. (12) can be seen in Fig. 4 where a similar simulation setup is adopted in the case of lognormal fading with S.I. $=0$.6. Results in Fig. 3 and Fig. 4 show that cooperation is more beneficial in the case of Rayleigh fading compared to lognormal fading where the performance gain can be realized at smaller error rates. This result is expected since the Rayleigh distribution is used to model the scenario of severe fading while the lognormal model corresponds to the scenario of less severe fading. The superiority of the cooperative scheme over non-cooperative direct FSO links can be also seen in Fig. 5 in the presence of background radiation. As in the no-background radiation case, gains are more significant over Rayleigh fading channels. While the assumption of channel independence can be justified in MIMO wireless RF systems, there is a wide agreement that this assumption is not often valid in MIMO-FSO systems and, consequently, the high gains promised by MIMO techniques might not be realized in practice. This point is investigated in Fig. 6 that compares the proposed cooperative system with the $2 \times 1$ MIMO-RC system in the presence of channel correlation. Results show that, for relatively large values of Es, the cooperative scheme shows approximately the same performance as the MIMO-RC system with a channel correlation factor of $\rho=$ 0.5 . The proposed scheme can even outperform MIMO-RC systems when $\rho=0.75$ or $\rho=0.9$.

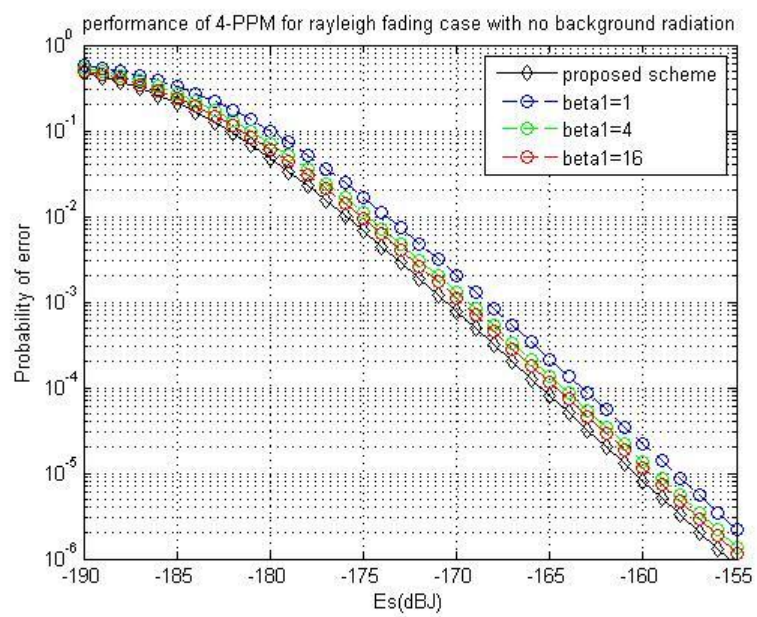

Figure :3

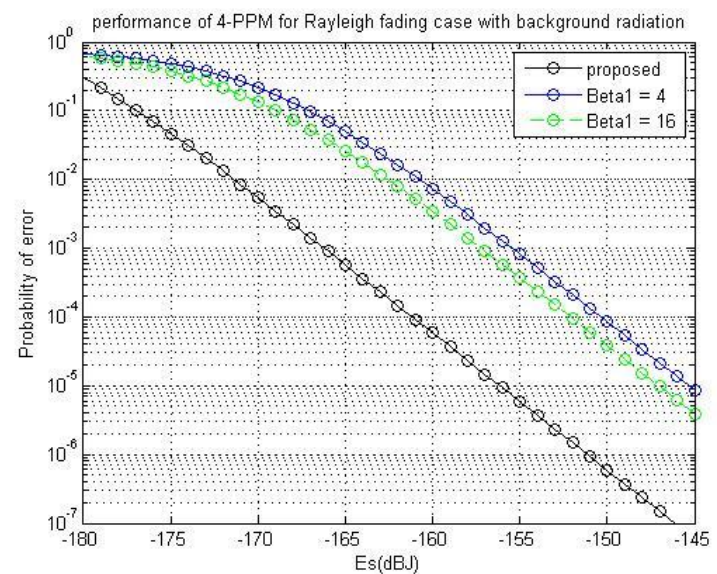

Figure $: 4$ 


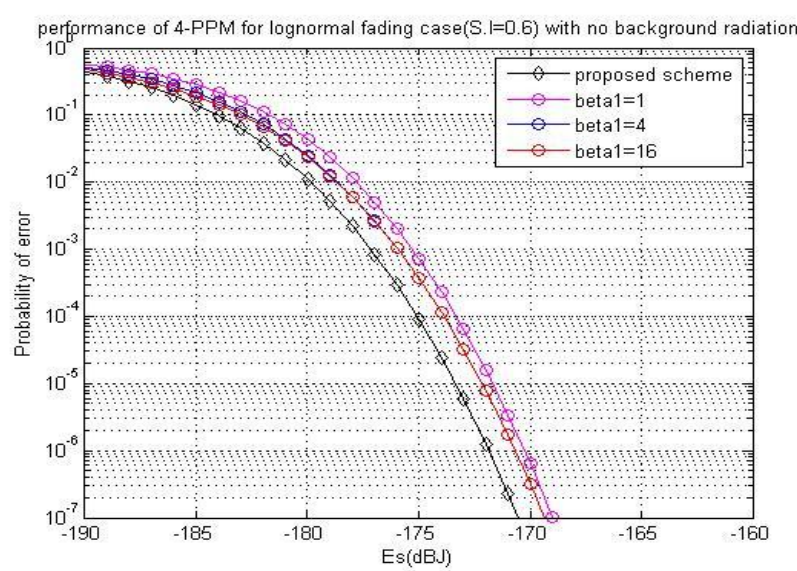

Figure :5

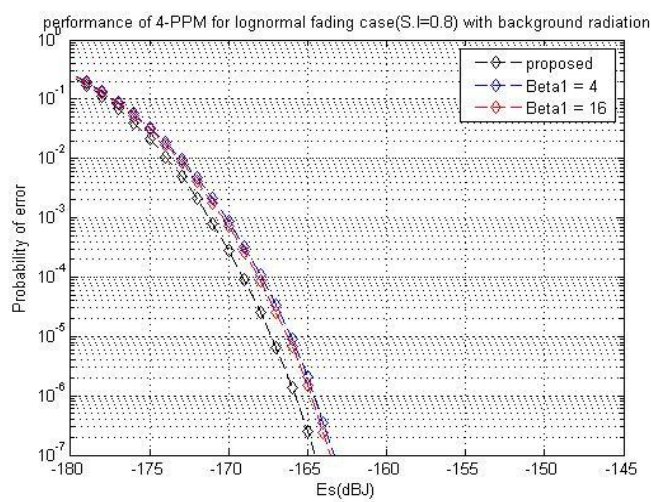

Figure:6

\section{Conclusion}

Despite the non-broadcast nature of FSO transmissions, this work showed that cooperative diversity can result in significant Performance gains over the non-cooperative $1 \times 1$ FSO links and over the $2 \times 1$ MIMOFSO links that suffer from correlated fading. It was proven analytically that a full transmit diversity order can be achieved in the no-background radiation case. In the presence of background radiation, a numerical integration of the conditional SEP showed that the proposed scheme can maintain acceptable performance gains especially in the case of Rayleigh fading. While this work analyzed cooperative diversity from a physical-layer point of view, future work must consider the implication of cooperation on higher layers.

\section{References}

[1] D. Kedar and S. Arnon, "Urban optical wireless communications networks: the main challenges and possible solutions," IEEE communication. Mag., vol. 42, no. 5, pp. 2-7, Feb. 2003.

[2] X. Zhu and J. Kahn, "Free-space optical communication through atmospheric turbulence channels," IEEE Transaction communication. vol. 50, no. 8, pp. 1293-1300, Aug. 2002.

[3] M.-A. Khalighi, N. Schwartz, N. Aitamer, and S. Bourennane, "Fading reduction by aperture averaging and spatial diversity in optical wireless systems," IEEE J. Optical communication. Network, vol. 1, pp. 580-593, Nov.2009.

[4] S. G. Wilson, M. Brandt-Pearce, Q. Cao, and J. H. Leveque, "Free-space optical MIMO transmission with Q-ary PPM," IEEE Trans. Communication., vol. 53, pp. 1402-1412, Aug. 2005.

[5] M. K. Simon and V. A. Vilnrotter, "Alamouti-type space-time coding for free-space optical communication with direct detection," IEEE Trans. Wireless communication. vol. 4, pp. 35-39, Jan. 2005.

[6] A. Garcia-Zambrana, C. Castillo-Vazquez, B. Castillo-Vazquez, and A. Hiniesta-Gomez, "Selection transmit diversity for FSO links over strong atmospheric turbulence channels," IEEE Photon. Technology Letter, vol. 21, pp. 1017-1019, July 2009.

[7] S. Navidpour, M. Uysal, and M. Kavehrad, "BER performance of free space optical transmission with spatial diversity," IEEE Trans. Wireless Communication, vol. 6, no. 8, pp. 2813-2819, Aug. 2007.

[8] T. Tsiftsis, H. Sandalidis, G. Karagiannidis, and M. Uysal, "Optical wireless links with spatial diversity over strong atmospheric turbulence channels," IEEE Trans. Wireless Communication, vol. 8, no. 2, pp. 951-957, Feb. 2009.

[9] J. Laneman and G. Wornell, "Distributed space time coded protocols for exploiting cooperative diversity in wireless networks," IEEE Transaction. Inf. Theory, vol. 49, no. 10, pp. 2415-2425, Oct. 2003.

[10] J. Laneman, D. Tse, and G. Wornell, "Cooperative diversity in wireless networks: efficient protocols and outage behavior," IEEE Trans. Inf. Theory, vol. 50, pp. 3062-3080, Dec. 2004.

[11] S. Halme, B. Levitt, and R. Orr, "Bounds and approximations for some integral expression involving lognormal statistics," MIT Res. Lab. Electron. Quart. Prog. Rept., 1969. 